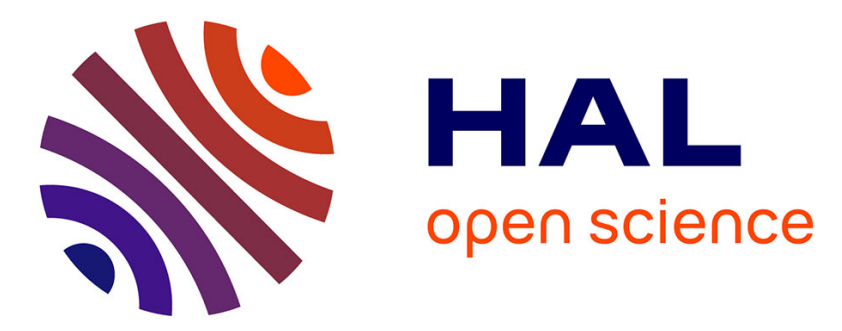

\title{
Observer Based Scheme for the Control of High Order Systems with Two Unstable Poles Plus Time Delay
}

David Fernando Novella Rodriguez, Basilio del Muro-Cuellar, Olivier Sename

\section{To cite this version:}

David Fernando Novella Rodriguez, Basilio del Muro-Cuellar, Olivier Sename. Observer Based Scheme for the Control of High Order Systems with Two Unstable Poles Plus Time Delay. Asia-Pacific Journal of Chemical Engineering, 2014, 9 (2), pp.167-180. 10.1002/apj.1757 . hal-01260386

\section{HAL Id: hal-01260386 \\ https://hal.science/hal-01260386}

Submitted on 22 Jan 2016

HAL is a multi-disciplinary open access archive for the deposit and dissemination of scientific research documents, whether they are published or not. The documents may come from teaching and research institutions in France or abroad, or from public or private research centers.
L'archive ouverte pluridisciplinaire HAL, est destinée au dépôt et à la diffusion de documents scientifiques de niveau recherche, publiés ou non, émanant des établissements d'enseignement et de recherche français ou étrangers, des laboratoires publics ou privés. 


\title{
Observer Based Scheme for the Control of High Order Systems with Two Unstable Poles Plus Time Delay
}

\author{
David Fernando Novella Rodríguez ${ }^{\mathrm{a}}$, Basilio Del Muro Cuéllar ${ }^{\mathrm{a}, *}$, Olivier \\ Sename ${ }^{b}$ \\ ${ }^{a}$ Escuela Superior de Ingeniería Mecánica y Eléctrica, Unidad Culhuacan, Instituto \\ Politécnico Nacional, Santa Ana 1000, México D.F., 04430, México \\ ${ }^{b}$ GIPSA-Lab Department of Control Systems, Grenoble INP, ENSE3, BP46 38402, Saint \\ Martin d'Hères Cedex, GRENOBLE, FRANCE.
}

\begin{abstract}
This paper deals with the problem of the stabilization and control of linear time invariant high order systems with two unstable real poles plus time delay. A simple observer based controller is designed in order to achieve a stable behavior of the closed loop system. Necessary and sufficient conditions for the existence of the proposed control structure are stated. Hence, only four proportional gains and the model of the plant are enough to obtain a stable response of the delayed system. Moreover, a robustness analysis is presented in order to compute the maximal uncertainty bound accepted for the delay term. In addition, a two degrees of freedom PI control action is implemented in order to track step references and to reject step disturbances. The achieved performance of the proposed control strategy is illustrated by mean of numerical simulations.
\end{abstract}

\section{Introduction}

Systems with significant delay term are very common, they appear in various systems as biological, ecological, social, industrial, etc., and are due to several mechanisms like material or energy transport, recycling loops, etc,. In addition, actuators, sensors and field networks that are involved in feedback loops usually introduce such delays, 1, 2]. Also delays can be used in model reduction where high-order (finite-dimensional) systems are approximated (in some norm sense) by low order systems with delays, [3]. It is known that time-delay is often a source of complex behaviors (oscillations, instability, bad performance), in many dynamic systems, and thus considerable attention has been paid on the stability analysis and controller design of time delay systems. Hence, there exists a great motivation to study delay effects on dynamical systems properties for two

\footnotetext{
${ }^{*}$ Corresponding author

Email addresses: dnovellar@gmail.com (David Fernando Novella Rodríguez), bdelmuro@yahoo.com (Basilio Del Muro Cuéllar), olivier.sename@gipsa-lab.grenoble-inp.fr (Olivier Sename)
} 
main reasons: first to understand how the delay presence may deteriorate the behavior of the system, and second to control their effects for better performance achievement on closed-loop systems, [4, 5].

Several control strategies have been developed to deal with delayed systems. A common approach is to approximate the time-delay operator by means of Taylor or Padé series which could lead to a non minimum-phase system with rational transfer function representation. This method can be successfully used only when the delay term is small compared with the dominant time constant of the plant.

A different approach to deal with dead time systems is the classical Smith Predictor (SP), which consists in counteracting the time delay effects by mean of strategies intended to estimate the effects of current inputs over future outputs, [6, 7]. The main limitation of the original SP is the fact that the prediction scheme does not have a stabilization step, which restricts its application to open-loop stable plants. However, open-loop unstable processes arise frequently in different dynamical systems and are fundamentally difficult to control. To overcome this problem, some modifications of the SP original structure have been proposed to deal with non-stable delayed process. For instance, [8] has presented an efficient modification to the Smith predictor in order to control unstable first order system plus time delay, in this case the stability bound is determined by the relationship $\tau / \tau_{u n}<1.5$, where $\tau$ is the process time delay and $\tau_{u n}$ the dominant unstable time-constant. With a different perspective, [9] proposes a modification to the original Smith structure in order to deal with unstable first order delayed systems. Using a similar structure, the result is extended to delayed high order systems in [10]. In both works, a robustness analysis is done concluding that for unstable dead time dominant systems, the closed-loop system can be unstabilized with an infinitesimal value of the modeling error, i.e., that robustness is strongly dependent on the relationship $\tau / \tau_{u n}$. For the control scheme proposed in this later work, it can be easily proven that in the case of unstable plants, the internal stability is not guaranteed. In fact it is obtained an unstable estimation error and, as a result, a minimal initial condition difference between the original plant and the model produces an internal unbounded signal.

The classical controllers Proportional-Integral-Derivative (P, PI, PID or PD) are also included between the tools used to deal with time delay systems. For instance, 11] introduce a study about the control of integral systems with dead time which includes the design and tune of PI and PID controllers by mean of different methods. In [12], the authors proposes a methodology to obtain a complete PI and PID parametrization for first order unstable delayed systems when $\tau<2 \tau_{u n}$. With a different perspective, in [13] upper bounds on the delay size $\left(\tau<2 \tau_{u n}\right)$ are provided when using linear time invariant controllers on the stabilization of strictly proper delayed real rational plants. It is important to note that in general, the provided bounds are not tight and the authors prevent that the developed controllers are not intended as practical solutions and are only used as a tool to compute the achievable delay margin for some particular cases. In [14], based in a numerical method, it is considered the stabilization of 
linear time-delay systems of order $n$. However, stability conditions with respect to time-delay and time constant of the process are not provided. The proposed method consists in shifting the unstable eigenvalues to the left half plane by static state feedback by applying small changes to the feedback gain. Using an observer based approach, in [15] a methodology is proposed in order to control unstable first order delayed systems allowing to deal with $\tau / \tau_{u n}<3$ and with a similar approach but including PID controllers in the loop, in [16] is stated a methodology to stabilize FOTD unstable Systems with $\tau / \tau_{u n}<4$.

The problem of proposing a controller scheme to deal with high order unstable systems, providing specific conditions on the delay size is more complex and they are not many works on this problem. For instance, in the work presented by Madhuranthakam et al., [17] it is proposed a method to the optimal tuning of PID controllers for first and second order systems plus time delay, nevertheless the method is restricted to stable processes. With a different perspective, [18] presents a strategy based on a modified smith predictor in order to control second order system but only the case of one unstable pole is dealt. On the other hand, [19] introduces a PID control strategy for second order unstable processes designed using the direct synthesis method. With a similar perspective, [20] presents a methodology in order to control second order delayed systems using the Internal Model Control guides for PID controllers. However, this works do not provide the stability bound in terms of the time delay size.

This paper is concerned with the stabilization problem of systems with two unstable poles and $m$ stable poles plus time delay. The control scheme relies on an observer-based structure with a memory observer and a memoryless state feedback, so only two gains are enough to stabilize the observer scheme and two other to stabilize the open loop unstable plant. Necessary and sufficient conditions are stated to guarantee the existence of the proposed scheme in terms of the relation between the size of the delayed term and the system time constants. On the contrary of modified Smith predictors, the scheme only contains discrete time delay (and not distributed ones) which makes easy its practical implementation (see [2] for details on numerical implementation of modified Smith predictor scheme). Moreover, it is implemented a Proportional-Integral controller which allows to track step references. It is worth stressing that, to the best of our knowledge, in the literature there is not reported similar results for this class of systems.

This paper is organized as follows; the Section 2 is dedicated to the problem formulation. The Section 3 yields the preliminaries results used to obtain the main result of this work. An observer based controller is proposed in the Section 4 in order to stabilize the unstable delayed system previously described, also the stability conditions of the proposed control structure are stated. In addition, it is illustrated how to deal with a zero in the left half plane in the delayed transfer function. Then, in Section 5 a robustness analysis for the observer based scheme with respect to the uncertainty in the delay operator is presented. Numerical simulations are presented in order to show the controller performance under different conditions in Section 6. Moreover, an application example related with a continuous stirred tank reactor (CSTR) is presented in order to refer the 
control strategy proposed with a practical framework. Finally some conclusions are drawn in Section 7.

\section{Problem Formulation}

Consider the following class of single-input single-output (SISO) linear systems with input delay:

$$
\frac{Y(s)}{U(s)}=\frac{N(s)}{D(s)} e^{-\tau s}=G(s) e^{-\tau s},
$$

where $U(s)$ and $Y(s)$ are the input and output signals respectively, $\tau \geq 0$ is the constant time delay, $N(s)$ and $D(s)$ are polynomials in the complex variable $s$ and $G(s)$ is the delay-free transfer function. Notice that with respect to the class of systems (11) a traditional control strategy based on an output feedback of the form:

$$
U(s)=C(s)[R(s)-Y(s)],
$$

yields a closed-loop system given by:

$$
\frac{Y(s)}{R(s)}=\frac{C(s) G(s) e^{-\tau s}}{1+C(s) G(s) e^{-\tau s}},
$$

where the exponential term $e^{-\tau s}$ located at the denominator of the transfer function (3) leads to a system with an infinite number of poles and where the closed-loop stability properties must be carefully stated.

This work proposes an observer based control scheme in order to stabilize a system characterized by the following transfer function:

$$
\frac{Y(s)}{U(s)}=\frac{\alpha}{(s-a)(s-b)\left(s+c_{1}\right)\left(s+c_{2}\right) \ldots\left(s+c_{m}\right)} e^{-\tau s} .
$$

Where $\tau \geq 0, a, b, c_{1}, c_{2} \ldots c_{m}>0$ are positive constants, and without loss of generality, $a \geq b>0$. The proposed control scheme has been designed taking into account the traditional observer theory hence only the plant model and two static gains are enough to get an adequate estimation of an internal delay free variable which will be used in the final stabilizing control scheme.

For sake of simplicity, we denote the stable subsystem $G_{s t b}(s)$ as follows

$$
G_{s t b}(s)=\frac{\alpha}{\left(s+c_{1}\right)\left(s+c_{2}\right) \ldots\left(s+c_{m}\right)} .
$$

\section{Preliminary Results}

Preliminary results are presented, which will be used later in order to state the stability conditions of the proposed strategy in this work.

Considering the following unstable first order system plus time delay: 


$$
\frac{Y(s)}{U(s)}=G(s) e^{-\tau s}=\frac{\alpha}{s-\sigma} e^{-\tau s},
$$

with $\sigma>0$, and a proportional output feedback control as follows:

$$
U(s)=R(s)-k Y(s),
$$

which produces a closed-loop system:

$$
\frac{Y(s)}{R(s)}=\frac{\alpha e^{-\tau s}}{s-\sigma+k \alpha e^{-\tau s}} .
$$

The following result has been widely studied in the literature and the proof can be easily obtained by considering different approaches as a classical frequency domain. An alternative simple proof based on a discrete time approach is shown in [21].

Lemma 1. Taking into account the delayed system (6) and the proportional output feedback (7). Then, there exists a proportional gain $k$ such that the closed loop system (8), is stable if and only if $\tau<\frac{1}{\sigma}$.

Now, take into consideration the high-order unstable system characterized by:

$$
G(s)=\frac{\alpha}{(s-\sigma)\left(s+\phi_{1}\right)\left(s+\phi_{2}\right) \ldots\left(s+\phi_{n}\right)} e^{-\tau s} .
$$

with $\sigma, \phi_{1}, \phi_{2} \ldots \phi_{n}>0$. With the proportional output feedback (7), the closed-loop system obtained is:

$$
\frac{Y(s)}{R(s)}=\frac{\alpha e^{-\tau s}}{\left[(s-\sigma)\left(s+\phi_{1}\right)\left(s+\phi_{2}\right) \ldots\left(s+\phi_{n}\right)\right]+k \alpha e^{-\tau s}} .
$$

Lemma 2. Considering the delayed system (9) and the proportional output feedback (7). Then, there exists a proportional gain $k$ such that the closed loop system (10), is stable if and only if $\tau<\frac{1}{\sigma}-\sum_{i=1}^{n} \frac{1}{\phi_{i}}$.

The proof of the previous lemma is presented in the Appendix A.

\section{Control Strategy Proposed}

Taking into account the class of systems studied in this work and characterized by the transfer function (4) with $a, b, c_{1}, c_{2}, \cdots, c_{m}>0$ and the time delay $\tau \geq 0$, and assuming without loss of generality $a \geq b$. An observer based control is designed in order to obtain an estimation of the internal states of the system to be used as control signals for the original process.

As a first step, the stability conditions for the controller and the observer systems are stated separately. This conditions will be used later in order to state the closed loop stability conditions for the proposed observer based controller. 


\subsection{Controller Scheme}

First, taking into consideration the controller structure shown in Figure 1 with the control law $u(t)=r(t)-k_{1} w(t)-k_{2} y(t)$, let us introduce the following result.

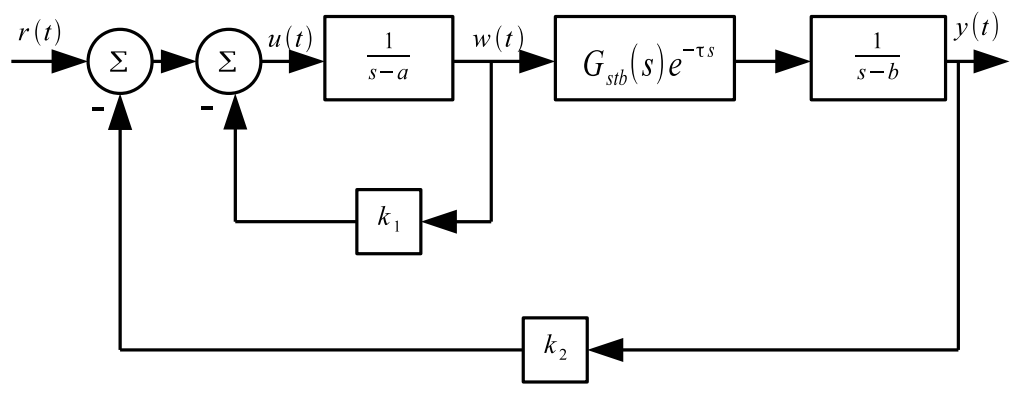

Figure 1: Control Scheme

Lemma 3. Consider the delayed system (4), and the control law mentioned above. There exist gains $k_{1}$ and $k_{2}$ such that the closed-loop system is stable if and only if

$$
\tau<\frac{1}{b}-\sum_{i=1}^{m} \frac{1}{c_{i}}
$$

The aim of the foregoing proof is to apply the stability conditions given in Lemma 2 to the state feedback strategy shown in the Figure 1.

Proof. Sufficiency. Let us consider $\tau<\frac{1}{b}-\sum_{i=1}^{m} \frac{1}{c_{i}}$. Then, $\tau=\frac{1}{b}-\sum_{i=1}^{m} \frac{1}{c_{i}}-\beta$, for some $\beta>0$. Therefore, there exists $k_{1}$ such that $\beta>\frac{1}{k_{1}-a}>0$. Then, it is easy to determine

$$
\tau<\frac{1}{b}-\sum_{i=1}^{m} \frac{1}{c_{i}}-\frac{1}{k_{1}-a} .
$$

Finally, we can conclude from Lemma2, where $\sigma=b, \phi_{i}=c_{i}$ and $\phi_{m+1}=k_{1}-a$ there exists $k_{2}$ such that the plant behavior is stable.

Necessity. Considering the delayed system (4), and the state feedback controller shown in Figure 3, with constant gains $k_{1}$ and $k_{2}$ such that the process is stable. The closed loop transfer function of the plant can be written as follows:

$$
\frac{Y(s)}{R(s)}=\frac{\alpha e^{-\tau s}}{(s-b)\left(s+c_{1}\right) \cdots\left(s+c_{m}\right)(s+\phi)+\alpha k_{2} e^{-\tau s}},
$$


with $\phi=k_{1}-a$. It is well known that a $k_{2}$ that stabilizes the delayed system (12) must also stabilize the delay free system (see for instance [5] or [22]), which implies that $\phi>0$. Indeed, from Lemma 2, $\tau<\frac{1}{\sigma}-\sum_{i=1}^{n} \frac{1}{\phi_{i}}$ with $\sigma=b$ and $\phi_{i}=c_{i}$, where $n=m+1$ (note that $\phi>0$ is a free parameter function of $k_{1}$ ). Let us consider $\beta>\frac{1}{\phi}>0$, denoting $\beta=\frac{1}{\sigma}-\sum_{i=1}^{m} \frac{1}{\phi_{i}}-\tau$, therefore:

$$
\tau=\frac{1}{\sigma}-\sum_{i=1}^{m} \frac{1}{\phi_{i}}-\beta<\frac{1}{\sigma}-\sum_{i=1}^{m} \frac{1}{\phi_{i}}=\frac{1}{b}-\sum_{i=1}^{m} \frac{1}{c_{i}}
$$

Remark 1. A simple methodology for choosing the values for the gains of the controller will be given below.

Step 1. In order to ensure the existence of a proportional gain $k_{2}$ such that the closed loop system is stable, from the proof of Lemma 3. eq. (11) we obtain

$$
k_{1}>\frac{1}{\frac{1}{b}-\sum_{i=1}^{m} \frac{1}{c_{i}}-\tau}+a .
$$

It is worth stress that if $k_{1}$ is selected near to the bound stated in (13), the gain margin for the gain $k_{2}$ will be reduced.

Step 2. Once a gain $k_{1}$ is selected, we can compute the gain $k_{2}$ by means of a frequency domain analysis, Nyquist stability criterion for instance, for the auxiliary system (12) such that the controller scheme shown in the Figure 1 is stable.

\subsection{Observer Scheme}

In most of the practical applications, the internal variables are not measured. Thus, an observer based on an output injection strategy is proposed, let us take into consideration the static output injection scheme shown in Figure 2, the stability of the observer can be tackled as follows.

Lemma 4. Considering the delayed system (4), and the static output injection scheme shown in Figure 2 . There exist constants $g_{1}$ and $g_{2}$ such that the closedloop system is stable if and only if

$$
\tau<\frac{1}{a}-\sum_{i=1}^{m} \frac{1}{c_{i}}
$$

Proof. The proof can be easily derived from a dual procedure of the previous result. 


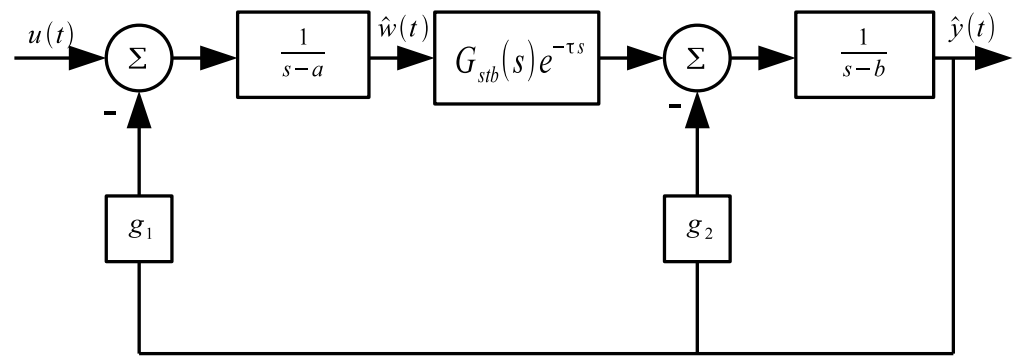

Figure 2: Observer Scheme.

Remark 2. As in the controller design, the computation of the gains $g_{1}$ and $g_{2}$ can be obtained in a dual way to Remark 1, i.e.,

Step 1. In order to ensure the existence of a proportional gain $g_{1}$ such that the closed loop system is stable, from Lemma 4 we obtain

$$
g_{2}>\frac{1}{\left(\frac{1}{a}-\sum_{i=1}^{m} \frac{1}{c_{i}}-\tau\right)}+b .
$$

Step 2. Once the gain $g_{2}$ is selected, we can compute the gain $g_{1}$ by means of a frequency domain analysis, Nyquist stability criterion for instance, such that the controller scheme shown in Figure 5 is stable.

\subsection{Observer-Based Controller: the case without zeros}

Finally, the main result of this work is presented, we propose an observed based controller as in the Figure 3, where the observer allows to estimate the state variables, to be used in state feedback controller. The authors would like to stress that, in the proposed scheme, only four proportional gains are enough to get a stable closed loop behavior. As a consequence of the previous results, the following lemma can be stated.

Lemma 5. Consider the observer based controller scheme shown in Figure 3. There exist proportional gains $k_{1}, k_{2}, g_{1}$ and $g_{2}$ such that the closed-loop system is stable if and only if

$$
\tau<\frac{1}{a}-\sum_{i=1}^{m} \frac{1}{c_{i}} .
$$

Proof. Consider a state space representation of the system (4) characterized by the following equation:

$$
\dot{x}(t)=A_{0} x(t)+A_{1} x(t-\tau)+B u(t)
$$




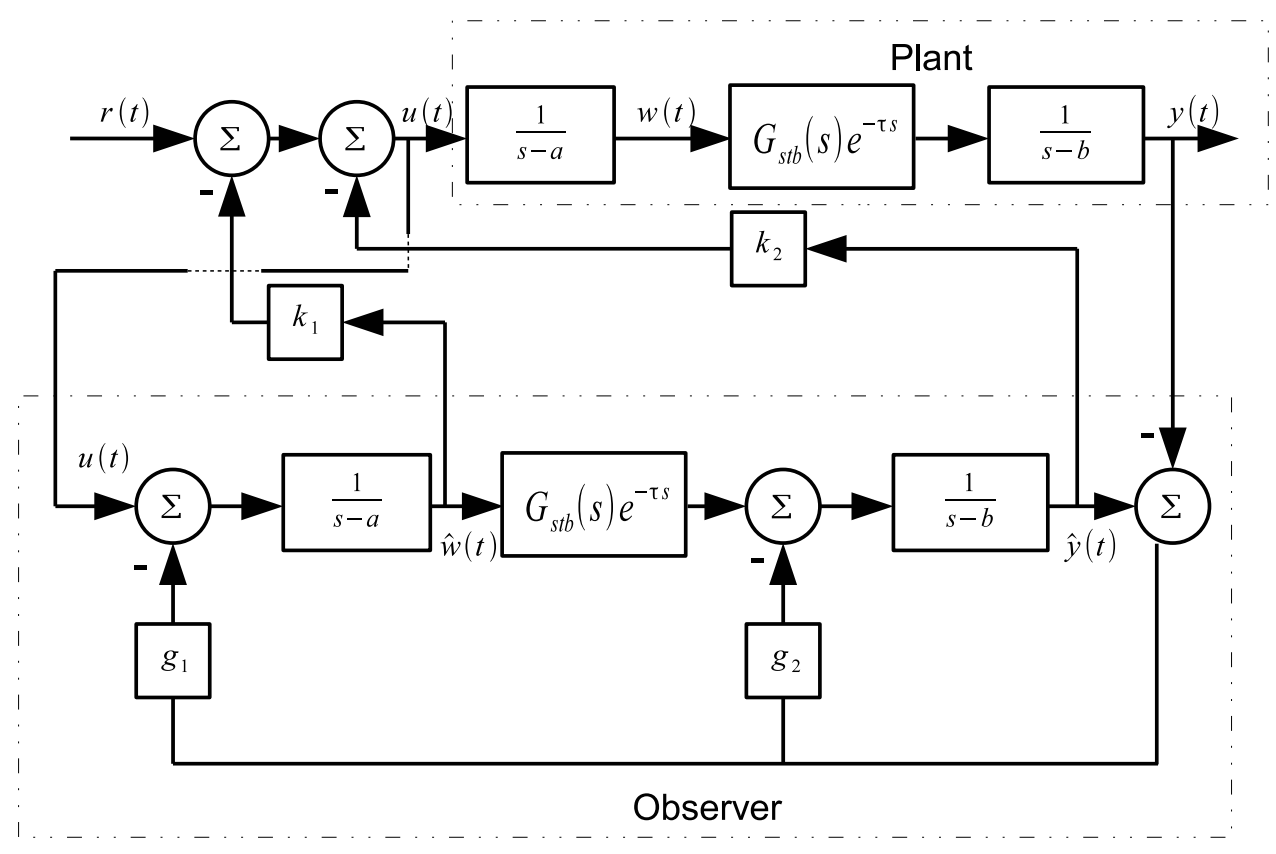

Figure 3: Control Strategy Proposed.

$$
y(t)=C x(t)
$$

with $x(t)=\left[\begin{array}{llllll}w(t) & x_{1}(t) & x_{2}(t) & \cdots & x_{m}(t) & z(t)\end{array}\right]^{T}$,

where the states $x_{i}(t)$ represent the stable part of the system, $w(t)$ and $z(t)$ are linked with the unstable poles of the open loop system. Then we have, 


$$
\begin{aligned}
A_{0}= & {\left[\begin{array}{ccccccc}
a & 0 & 0 & \cdots & 0 & 0 & 0 \\
1 & -c_{1} & 0 & \cdots & 0 & 0 & 0 \\
0 & 1 & -c_{2} & \cdots & 0 & 0 & 0 \\
\vdots & \vdots & \vdots & \ddots & \vdots & \vdots & \vdots \\
0 & 0 & 0 & \cdots & 1 & -c_{m} & 0 \\
0 & 0 & 0 & \cdots & 0 & 0 & b
\end{array}\right], } \\
A_{1}= & {\left[\begin{array}{ccccccc}
0 & 0 & 0 & \cdots & 0 & 0 & 0 \\
0 & 0 & 0 & \cdots & 0 & 0 & 0 \\
0 & 0 & 0 & \cdots & 0 & 0 & 0 \\
\vdots & \vdots & \vdots & \ddots & \vdots & \vdots & \vdots \\
0 & 0 & 0 & \cdots & 0 & 0 & 0 \\
0 & 0 & 0 & \cdots & 0 & 1 & 0
\end{array}\right] } \\
B= & {\left[\begin{array}{c}
\alpha \\
0 \\
0 \\
\vdots \\
0 \\
0
\end{array}\right], }
\end{aligned}
$$

Note that the state state representation characterized by (14) can be returned to its transfer function representation by mean of:

$$
\frac{Y(s)}{U(s)}=C\left(s I-\left(A_{0}+A_{1} e^{-\tau s}\right)\right)^{-1} B,
$$

which brings us back to the delayed transfer function (4). The dynamics of the estimated states and the control law can be described as follows.

$$
\dot{\hat{x}}(t)=A_{0} \hat{x}(t)+A_{1} \hat{x}(t-\tau)+B u(t)-G(C \hat{x}(t)-y(t))
$$

Where $\hat{x}(t)$ is the estimated state of $x(t)$, and the gain vectors $K$ and $G$ are defined by

$$
\begin{aligned}
K & =\left[\begin{array}{llllll}
k_{1} & 0 & 0 & \cdots & 0 & k_{2}
\end{array}\right], \\
G & =\left[\begin{array}{llllll}
g_{1} & 0 & 0 & \cdots & 0 & g_{2}
\end{array}\right]^{T} .
\end{aligned}
$$

Let $e(t):=x(t)-\hat{x}(t)$, then we have:

$$
\dot{e}(t)=\dot{x}(t)-\dot{\hat{x}}(t)=\left(A_{0}-G C\right) e(t)+A_{1} e(t-\tau),
$$

and the controlled system: 


$$
\dot{x}(t)=A_{0} x(t)+A_{1} x(t-\tau)-B K \hat{x}(t) .
$$

Noting $x_{e}=[x(t) e(t)]^{T}$ and after a simple manipulation of variables we have the following closed loop system with the observer and the controller proposed in the Figure 3

$$
\begin{gathered}
\dot{x_{e}}(t)=\left[\begin{array}{cc}
A_{0}-B K & B K \\
0 & A_{0}-G C
\end{array}\right] x_{e}(t)+\left[\begin{array}{cc}
A_{1} & 0 \\
0 & A_{1}
\end{array}\right] x_{e}(t-\tau) \\
y(t)=\left[\begin{array}{ll}
C & 0
\end{array}\right] x_{e}(t) .
\end{gathered}
$$

It is easy to see that the observer based controller proposed satisfies the separation principle. Hence, the stability of the observer scheme is enough to assure an adequate error convergence, i.e. there exist proportional gains $g_{1}$ and $g_{2}$ such that $\lim _{t \rightarrow \infty}[\hat{w}(t)-w(t)]=0$ if and only if

$$
\tau<\frac{1}{a}-\sum_{i=1}^{m} \frac{1}{c_{i}}
$$

then, considering the fact of the observer and controller can be designed separately and reminding the stability conditions stated previously in Lemmas 3 and 4, is clear that the observer stability condition is more restrictive than the controller one, i.e.,

$$
\frac{1}{a}-\sum_{i=1}^{m} c_{i}<\frac{1}{b}-\sum_{i=1}^{m} \frac{1}{c_{i}}
$$

therefore, there exist $k_{1}, k_{2}, g_{1}$ and $g_{2}$ such that the closed-loop system is stable if and only if

$$
\tau<\frac{1}{a}-\sum_{i=1}^{m} \frac{1}{c_{i}}
$$

Remark 3. Different works have been presented recently in order to obtain a maximum upper bound for the time delay. For instance, in [13] the authors adopt a frequency domain approach to demonstrate that, for a strictly proper rational plant, there exists an uniform upper bound on the delay that can be tolerated when using a finite dimensional linear time invariant (FDLTI) controller. Thus, for plants with an unstable pole $p$, the maximum margin delay obtained is $D M \leq 2 / p$. Furthermore, the authors consider a controller (see [13], Remark 8) to achieve this delay margin, although this class of controller may suffer difficulties such poor sensitivity, phase margin, etc., therefore, it is not intended as a practical controller. Also, the authors have the assumption that, for a plant with two unstable poles (the class of systems dealt in this work) the delay margin of the system is $D M \leq \min \left\{2 / p_{1}, 2 / p_{2}\right\}$, where $p_{1}$ and $p_{2}$ are the unstable poles of the system. However the authors do not propose a controller in order to obtain this delay margin. 


\subsection{Observer-Based Controller: the case with a left half plane zero}

The proposed control strategy can be easily implemented when the system contains a left half plane zero. For this, the main idea is to cancel the zero dynamics for each case (controller and observer), placing an unstable pole in the position of the zero. For this consider the stable subsystem $G_{s t b}(s)$ given by the following transfer function

$$
G_{s t b}(s)=\frac{\alpha(s+\beta)}{\left(s+c_{1}\right)\left(s+c_{2}\right) \ldots\left(s+c_{m}\right)} .
$$

Remark 4. As was mentioned before, the main idea to deal with the case of systems with a left half plane zero is to cancel the zero dynamics by means the relocation of an unstable pole, from this we can state the following procedure to obtain the gains for the observer based structure in this case.

Step 1. In order to cancel the zero dynamics by means of the selection of $k_{1}$ in the controller scheme, we have the following equality

$$
k_{1}=\beta+a
$$

Step 2. From this, by means of a frequency domain analysis, Nyquist stability criterion for instance, we can compute the gain $k_{2}$ for the obtained system

$$
\frac{Y(s)}{R(s)}=\frac{\alpha e^{-\tau s}}{(s-b)\left(s+c_{1}\right) \cdots\left(s+c_{m}\right)},
$$

Step 3. In order to cancel the zero dynamics by means of the selection of $g_{2}$ in the observer scheme, we have the following equality

$$
g_{2}=\beta+b
$$

Step 4. From this, by means of a frequency domain analysis, Nyquist stability criterion for instance, we can compute the gain $g_{1}$ for the obtained system

$$
\frac{Y(s)}{R(s)}=\frac{\alpha e^{-\tau s}}{(s-a)\left(s+c_{1}\right) \cdots\left(s+c_{m}\right)},
$$

\subsection{Step tracking reference}

In order to solve the step tracking reference problem, the observed based structure can be complemented with a proportional-integral (PI) control action, shown in Figure 4. For high-order time delay systems, a PI controller design is proposed in 23]. As it is well known, conventional methods of PI controllers design involve a zero on the closed loop system which yields an undesirable

overshoot on the response. Therefore, a two degrees of freedom PI controller will be considered to overcome this undesirable overshot response, [1], 24]. The PI controller is characterized as follows 


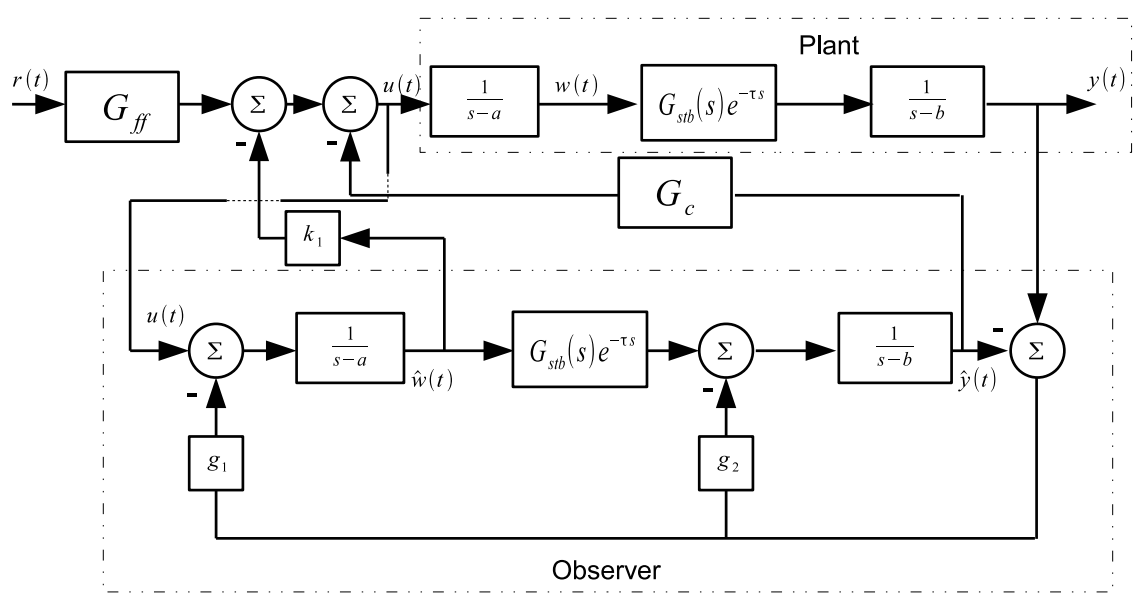

Figure 4: Control Structure

$$
U(s)=G_{f f}(s) R(s)-G_{c}(s) \hat{Y}(s)
$$

where

$$
G_{c}(s)=K_{p}\left(1+\frac{K_{I}}{s}\right)
$$

and

$$
G_{f f}(s)=K_{p}\left(\epsilon+\frac{K_{I}}{s}\right) .
$$

Here, the main idea is to relocate the unstable pole $a$ by means of the gain $k_{1}$, then we have a open loop process with an unstable pole located at $s=b$ and $m+1$ stable poles, characterized by the equation (12). Then it is possible to use the methodology given in 23] in order to tune a PI controller. We can resume the procedure as follows (for more details see [23]); first the gain $K_{I}$ must be selected such that $\Phi(\omega)>-\pi$, for some $w^{\star}>0$, with the function

$$
\Phi(\omega)=-\tau \omega-\pi+\arctan \left(\frac{\omega}{b}\right)-\arctan \left(\frac{K_{I}}{\omega}\right)-\sum_{i=1}^{m+1} \arctan \left(\frac{\omega}{c_{i}}\right) .
$$

Then, the range of stabilizing gains $K_{p}$ is given by

$$
\sqrt{\frac{\left(\omega_{c 1}^{2}+b^{2}\right) \prod_{i=1}^{m}+1\left(\omega_{c 1}^{2}+c_{i}^{2}\right)}{1+\left(\frac{K_{I}}{\omega_{c 1}}\right)}}<K_{p}<\sqrt{\frac{\left(\omega_{c 2}^{2}+b^{2}\right) \prod_{i=1}^{m}+1\left(\omega_{c 2}^{2}+c_{i}^{2}\right)}{1+\left(\frac{K_{I}}{\omega_{c 2}}\right)}}
$$

with $\omega_{c 1}<\omega_{c 2}$ being the first two phase frequencies solved from 


$$
-\tau \omega+\arctan \left(\frac{\omega}{b}\right)-\arctan \left(\frac{K_{I}}{\omega}\right)-\sum_{i=1}^{m+1} \arctan \left(\frac{\omega}{c_{i}}\right)=0 .
$$

Then, in order to reduce the undesirable overshoot in the response, the parameter $\epsilon$ can be chosen from $0<\epsilon<1$.

\section{Robustness Analysis With Respect To Delay Uncertainty.}

Here, we refer to robustness with respect to delay to the fact that the exact value of the delay is not known a priori. Therefore, we assume now that the delay is uncertain, $i$. $e$. the delay of the real system is $\tau=d(1+\delta \theta)$ and may be different from the one used in the observer (the nominal delay $d$ ). Consider the delay uncertainty in the original process, and the design of the observer taking into account the nominal time delay, i.e.

$$
\begin{aligned}
\dot{x}(t) & =A_{0} x(t)+A_{1} x(t-\tau)+B u(t) \\
\dot{\hat{x}}(t) & =A \hat{x}(t)+A_{1} \hat{x}(t-d)+B u(t)-G(C \hat{x}(t)-y(t)) \\
u(t) & =-K \hat{x}(t)
\end{aligned}
$$

Let us consider the observer-controller system with the form (29) and its extended closed loop system considering the delay uncertainty characterized as follows.

$$
\begin{aligned}
\dot{x_{e}}(t)= & {\left[\begin{array}{cc}
A_{0}-B K & B K \\
0 & A_{0}-G C
\end{array}\right] x_{e}(t)+\left[\begin{array}{cc}
A_{1} & 0 \\
A_{1} & 0
\end{array}\right]\left(x_{e}\right)_{\tau}(t-\tau)+\cdots } \\
\cdots+ & {\left[\begin{array}{cc}
0 & 0 \\
-A_{1} & A_{1}
\end{array}\right]\left(x_{e}\right)_{d}(t-d) } \\
& =\mathcal{A}_{0} x_{e}(t)+\mathcal{A}_{\tau}\left(x_{e}\right)_{\tau}(t-\tau)+\mathcal{A}_{d}\left(x_{e}\right)_{d}(t-d)
\end{aligned}
$$

Note that when the delay value of the actual process matches to the nominal one, the extended closed loop system (31) is equivalent to the nominal closed loop system (19), 25]. Now modeling the time delay with a multiplicative uncertainty as $\tau=d(1+\delta \theta)$, with $\theta$ normalized, i.e. $|\theta| \leq 1$ we obtain.

$$
e^{-s \tau}=e^{-s d(1+\delta \theta)}=e^{-s d} e^{-s d \delta \theta}=e^{-s d}(1-\Delta)
$$

With $\Delta(s)=1-e^{-s d \delta \theta}$. Therefore the characteristic polynomial of the above system is given by:

$$
\Psi(s)=\operatorname{det}\left[\Psi_{0}(s)\right] \operatorname{det}\left[I_{n}+\Psi_{0}^{-1}(s) \mathcal{A}_{\tau} e^{-s d} \Delta(s)\right]
$$

Where $\Psi_{0}(s)=I_{2 n}-\mathcal{A}_{0}-\left(\mathcal{A}_{\tau}+\mathcal{A}_{d}\right) e^{-s d}$. Now, when the design of the controller and the observer ensures that the nominal extended system is stable, 
i. e. $\operatorname{det}\left[\Psi_{0}(s)\right]$ is stable, the perturbed closed loop system remains stable if $\operatorname{det}\left[I_{n}+\Psi_{0}^{-1}(s) \mathcal{A}_{\tau} e^{-s d} \Delta(s)\right]$ does not change sign when $s$ sweeps the imaginary axis. Invoking Rouche's Theorem, it follows that the condition for stability is:

$$
\left\|Q_{d}(s) \Delta(s)\right\|_{\infty}<1
$$

Where $Q_{d}(s)=\Psi_{0}^{-1}(s) \mathcal{A}_{\tau} e^{-s d}$. Note that the term $1-e^{-s d \delta \theta}=\left.s d \delta \theta e^{-s d \delta \theta / 2}\right|_{s=j \omega}$ $\operatorname{sinc} \frac{\omega d \delta \theta}{2 \pi}$. Hence, on the imaginary axis, $\left|1-e^{-s d \delta \theta}\right| \leq|s d \delta \theta|$, then if one can show stability for $1-e^{s d \delta \theta}$ replacing it by $s d \delta \theta$ in the analysis, then the stability for the uncertain system will follow. Note that this amounts to replacing the delayed term by its first order Taylor expansion. This means that the maximal uncertainty bound $\delta$ that preserves stability for the worst case, i.e. $\theta=1$, is determined by:

$$
\delta_{\max }=\frac{1}{\left\|s d e^{-s d} \Psi_{0}^{-1}(s) \mathcal{A}_{\tau}\right\|_{\infty}}
$$

Then for all $|\theta| \leq 1$ and the uncertainty bound $\delta_{\max }$ the determinant has a fixed sign, implying the absence of zero crossings, and henceforth the stability of the perturbed system (provided the nominal one is stable) [25, 26].

Remark 5. Note that the stabilizing gains computed for the observer based controller for the nominal time delay $d$ are able to preserve stability of the closed loop system with a nominal delay belongs to the interval $\left[\begin{array}{ll}0 & d\end{array}\right]$, the analysis of the stability sets and regions are dealt in [5] and [22]

\section{Examples}

Below, numeric simulations are performed in order to illustrate the observer based scheme performance. Moreover, the PI control action is implemented to achieve step reference tracking and step disturbance rejection.

Example 1. Consider the fourth order delayed system with two unstable poles characterized with the following transfer function:

$$
\frac{Y(s)}{U(s)}=\frac{5}{(s-0.7)(s-0.4)(s+5)(s+10)} e^{-s}
$$

Let be $a=0.7, b=0.4, c_{1}=5, c_{2}=10, \alpha=1$ and $\tau=1$ the parameters of the system, it is clear that the stability condition given in Lemma 5 is satisfied, therefore there exists an observer based structure with proportional gains $k_{1}, k_{2}, g_{1}$ and $g_{2}$ such that the resulting closed-loop system is stable due to:

$$
\tau=1<\frac{1}{a}-\sum_{i=1}^{m} \frac{1}{c_{i}}=1.128 .
$$

Following the methodology given in remarks 1 and 2, the stability regions for the stabilizing gains are presented in Figure 5] from this the selected values for the control structure are shown in Table 1 Figure 6] illustrate the output 
performance of the observer based controller in numerical simulations. The continuous line indicates the output of the closed loop system with identical initial conditions between $y(t)$ and $\hat{y}(t)$. The dashed line point to the system performance whit different initial conditions, $(\hat{y}(0)-y(0)=0.01)$.

\begin{tabular}{|c|c|}
\hline$k_{1}$ & 10.7 \\
\hline$k_{2}$ & 55 \\
\hline$g_{1}$ & 145 \\
\hline$g_{2}$ & 20.4 \\
\hline
\end{tabular}

Table 1: Observer Based Controller Gains: Example 1.
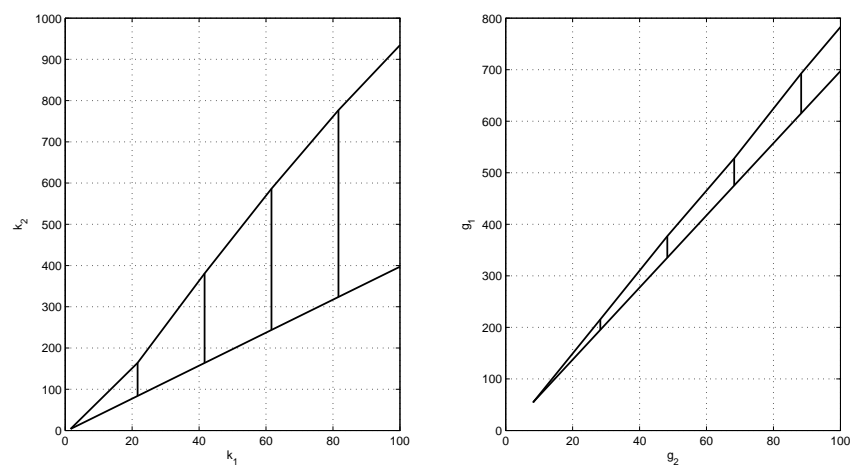

Figure 5: Stability regions for proportional gains in the observer based controller

Remark 6. As can be shown in Figure 5, the selection of a larger value of $k_{1}$, (resp. $g_{2}$ ), gives a larger gain margin for $k_{2}$, (resp. $\left.g_{1}\right)$. However, a selection of high gains for the observer based controller could give the problem of high control signals.

Remark 7. It is possible to implement a similar controller as the presented in [13] (Remark 8) in order to obtain better stability conditions for the control strategy proposed in this work, however some properties of the system (phase margin, gain margin, etc.) can be affected.

\section{Robustness Analysis}

The Figure 7 shown the output signal of the closed loop system with different initial conditions between the plant and the observer system, $(\hat{y}(t)-y(t)=0.01)$, when there is not uncertainty in the delay value (continuous line), and the closed loop system behavior when the maximum uncertainty bound is presented in the system (dashed line). The following table shows the relation between the size of the time delay $d$ of the nominal system and the maximal uncertainty bound 

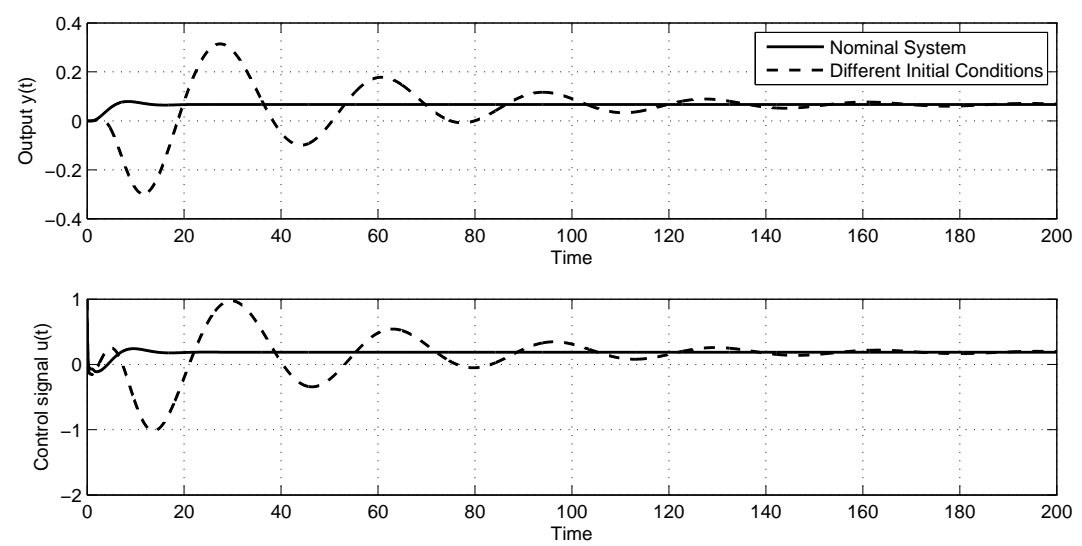

Figure 6: Numerical Simulation Results.

$\delta_{\max }$ in the above example. The terms $\tau_{\min }$ and $\tau_{\max }$ indicate the minimal and maximal value respectively of the delay term $\tau$, such that the closed loop system with nominal delay $d$ remains stable.
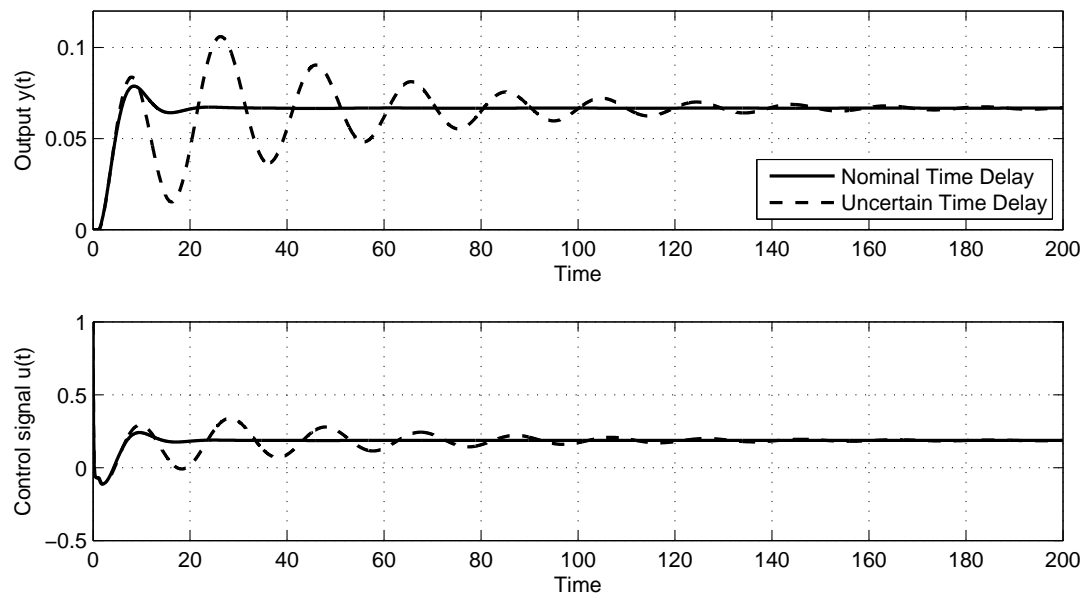

Figure 7: Robustness Analysis with respect to Time Delay

Example 2. Chemical reactors often have significant heat effects, so it is important to be able to add or remove heat from them. In a jacketed CSTR (continuously stirred tank reactor) the heat is added or removed by virtue of the temperature difference between a jacket fluid and the reactor fluid. Often, the heat transfer fluid is pumped through agitation nozzles that circulate the 


\begin{tabular}{|c|c|c|c|}
\hline$d$ & $\delta_{\max }$ & $\tau_{\min }$ & $\tau_{\max }$ \\
\hline 0.1 & 0.593279 & 0.040672 & 0.159328 \\
\hline 0.2 & 0.286649 & 0.142670 & 0.257330 \\
\hline 0.3 & 0.184523 & 0.244643 & 0.355357 \\
\hline 0.4 & 0.133386 & 0.346646 & 0.453354 \\
\hline 0.5 & 0.102516 & 0.448742 & 0.551258 \\
\hline 0.6 & 0.081652 & 0.551009 & 0.648991 \\
\hline 0.7 & 0.066381 & 0.653533 & 0.746467 \\
\hline 0.8 & 0.054500 & 0.756400 & 0.843600 \\
\hline 0.9 & 0.044822 & 0.859660 & 0.940340 \\
\hline 1 & 0.036679 & 0.963321 & 1.036679 \\
\hline
\end{tabular}

Table 2: Robustness Analysis with different time delay size.

fluid through the jacket at high velocity, as is shown in Figure 8 ,

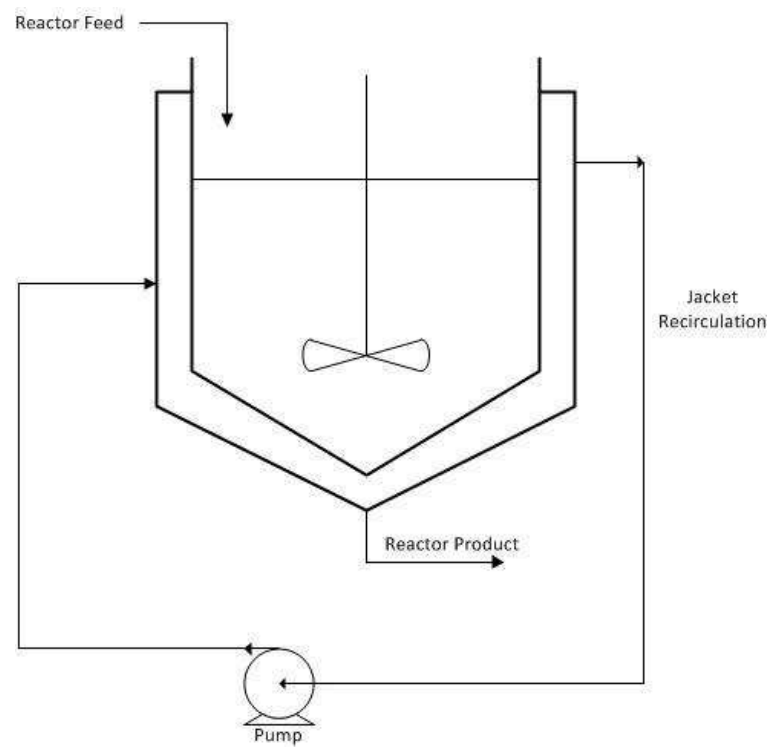

Figure 8: Continuos Stirred Tank Reactor

Here we consider a CSTR carrying out the simple reaction $A \rightarrow B$. The balance on component $A$ is

$$
V \frac{d C_{A}}{d t}=F C_{A f}-F C_{A}-V r_{A},
$$

where $C_{A}$ is the concentration of component $A$ in the reactor and $r_{A}$ is the rate of reaction per unit volume. The Arrhenius expression is normally used for the rate of reaction. A first order reaction results in the following 


$$
r_{A}=k_{0} e^{\left(\frac{-\Delta E}{R T} C_{A}\right)},
$$

where $k_{0}$ is the frequency factor, $E_{a}$ is the activation energy, $R$ is the ideal gas constant, and $T$ is the reactor temperature on an absolute scale. A detailed model of a CSTR includes the effect of cooling jacket dynamics. Then, the three modelling equations are

$$
\begin{aligned}
\frac{d C_{A}}{d t} & =\frac{F}{V}\left(C_{A f}-C_{A}\right)-k_{0} e^{\left(\frac{-\Delta E}{R T}\right)} C_{A}, \\
\frac{d T}{d t} & =\frac{F}{V}\left(T_{f}-T\right)+\frac{-\Delta H}{R T} k_{0} e^{\left(\frac{-\Delta E}{R T}\right)} C_{A}-\frac{U A}{V \rho c_{p}}\left(T-T_{j}\right), \\
\frac{d T_{j}}{d t} & =\frac{F_{j f}}{V_{j}}\left(T_{j f}-T_{j}\right)+\frac{U A}{V_{j} \rho_{j} c_{p j}}\left(T-T_{j}\right) .
\end{aligned}
$$

Where where $-\Delta H$ is the heat of the reaction, $U$ is the heat transfer coefficient, $A$ is the heat transfer area, $T_{f}$ is the feed temperature, $T_{j}$ is the jacket temperature, $T_{j f}$ is the jacket feed temperature and $F_{j f}$ the jacket make-up flow rate, [27].

The parameter values of the system are given as $\Delta E=20000 \mathrm{Btu} / \mathrm{lbmol}$, $k_{0}=16.96 \times 10^{7} \mathrm{hr}^{-1},-\Delta H=19000 \mathrm{Btu} / \mathrm{lbmol}, \mathrm{U}=75 \mathrm{Btu} / \mathrm{hr}{ }^{\circ} \mathrm{Fft} \mathrm{t}^{2}, \rho c_{p}=$ $53.25 \mathrm{Btu} /{ }^{\circ} \mathrm{Fft} t^{3}, \rho_{j} c_{p j}=55.6 \mathrm{Btu} /{ }^{\circ} \mathrm{Fft} \mathrm{t}^{3}$. The operating values of the reactor are given by $A=88 \mathrm{ft}^{2}, C_{A f}=0.132 \mathrm{lbmol} / \mathrm{ft}^{3}, T_{f}=60^{\circ} \mathrm{F}$, the operating volume $V=85 \mathrm{ft}^{3}$, the flow rate $F=35 \mathrm{ft}^{3} / \mathrm{hr}$, the jacket volume $V_{j}=25 f^{3}$, the jacket temperature $T_{j f}=0^{\circ} \mathrm{F}$ and the jacket make-up flow rate is $F_{j f}=28.75 \mathrm{ft}^{3} / \mathrm{hr}$. A steady state operating point is $C_{A}=0.166 \mathrm{lbmol} / \mathrm{ft}^{3}$ and $T=101.1^{\circ} \mathrm{F}$. Let consider the flow rate as the manipulated variable and the temperature of the CSTR as the controlled variable. Linearisation around this steady state operating point yields the following transfer function model (by assuming a measurement time delay of 54 minutes)

$$
\frac{T(s)}{F_{j}(s)}=\frac{-4.7475(s+3.129)}{(s-0.5545)(s-0.09395)(s+6.394)} e^{-0.9 s}
$$

Figure 9 illustrates the output response of the closed loop system stabilized by means of the observer-based controller for a unit step reference in numerical simulations, the computed gains are shown in Table 3. Continuous line denotes the nominal system, dashed line denotes the closed loop system with an uncertainty of $10 \%$ in the real process.

For the present example, following the methodology proposed in [23], the parameters computed for the PI controller are shown in Table 3 and for the feed forward compensator $G_{f f}$, the set-point weighting parameter $\epsilon=0.01$. The proportional gains $k_{1}, g_{1}$ and $g_{2}$ are the same that the computed before. The closed loop response is shown in Figure 10, continuous line shows the performance of the observer based controller with the complement of the PI control action considering the nominal plant. A step disturbance with magnitude $d=0.01$ is 

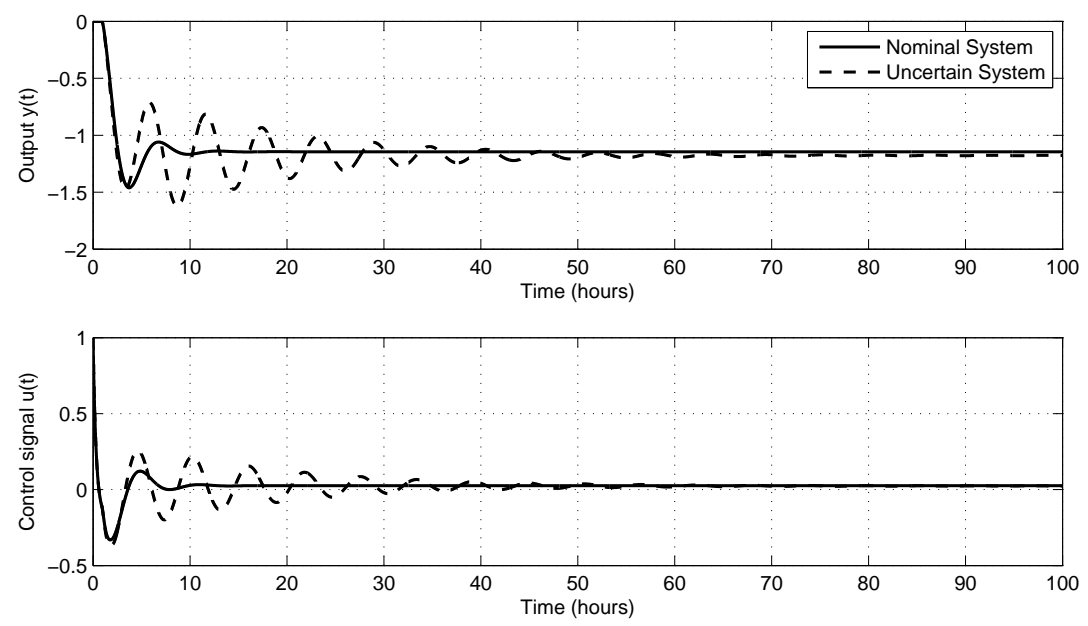

Figure 9: Control Strategy Performance

applied at $t=50 h r$, as it can be noticed, also the PI controller is able to reject step disturbances. The dashed line point to the behavior for the closed loop system with different initial conditions in the observer scheme $(y(t)-\hat{y}(t)=0.2)$.

\begin{tabular}{|c|c|}
\hline$k_{1}$ & 3.6835 \\
\hline$k_{2}$ & -1 \\
\hline$g_{1}$ & -1.2 \\
\hline$g_{2}$ & 3.2230 \\
\hline$K_{p}$ & -0.6 \\
\hline$K_{I}$ & 0.2774 \\
\hline
\end{tabular}

Table 3: Observer Based Controller Gains: Example 2.

Remark 8. Numerical simulations above show an efficient performance of the control strategy proposed even in face of different initial conditions as well as parametric uncertainties in the system.

Example 3. The following example is presented in [19], it is a second order system plus time delay (a particular case of the class of systems dealt in this work) with the transfer function given by

$$
\frac{Y(s)}{U(s)}=\frac{2}{(3 s-1)(s-1)} e^{-\tau s}
$$

For this example, the parameters of the system are $a=1, b=0.333, \alpha=$ 0.6667 and $\tau=0.3$. The gains computed for the observer and the PI controller 

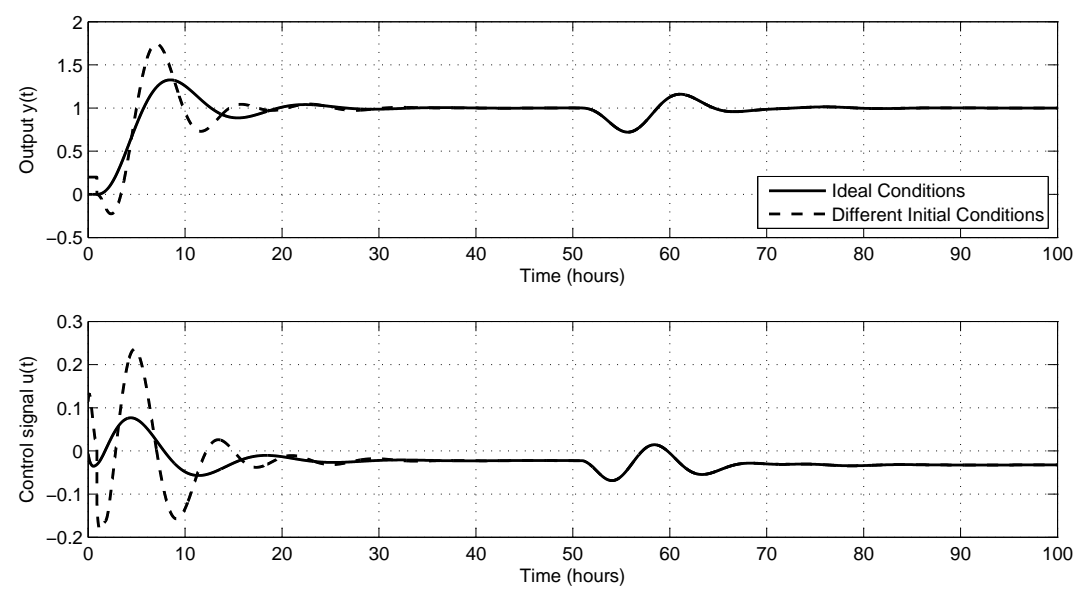

Figure 10: Step Tracking Performance

are shown in Table 4. Figure 11 shows the response of the system of example 3. A step negative disturbance is presented in $t=30$. Solid line point the performance for the control strategy proposed in this work and dashed line shows the performance of the methodology proposed in [19]. It can be noticed that the observer based structure proposed in this work gives a better performance that the PID controller proposed in [19]. In addition, the methodology proposed in [19] does not state bounds in terms of the time delay size, while our control structure can be implemented in the system with a larger delay as it is shown in Figure 12. The system of Example 3 is controlled with the observer based controller proposed, but with a time delay $\tau=0.8$. The computed parameters of the observer structure and the PI controller are shown in Table 4

\begin{tabular}{|c|c|c|}
\hline & $\tau=0.3$ & $\tau=0.8$ \\
\hline$k_{1}$ & 151.5 & 76.5 \\
\hline$g_{1}$ & 250 & 520 \\
\hline$g_{2}$ & 50.33 & 320.33 \\
\hline$K_{p}$ & 200 & 90 \\
\hline$K_{I}$ & 0.3 & 0.25 \\
\hline
\end{tabular}

Table 4: Observer Based Controller Gains: Example 3.

\section{Conclusions}

An observer based controller is proposed in order to stabilize high order system with two unstable poles plus time delay. The necessary and sufficient conditions that ensure the existence of the stabilizing controller scheme are stated. 


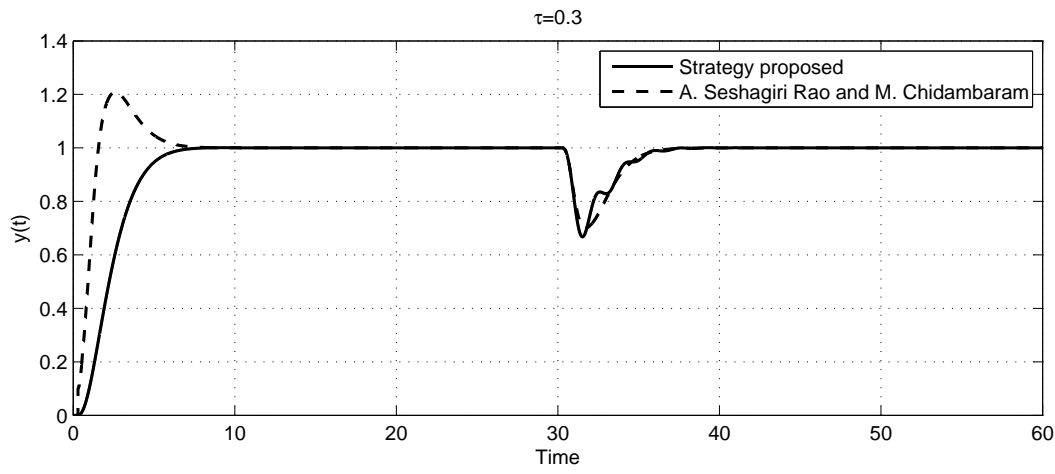

Figure 11: Performance Comparison

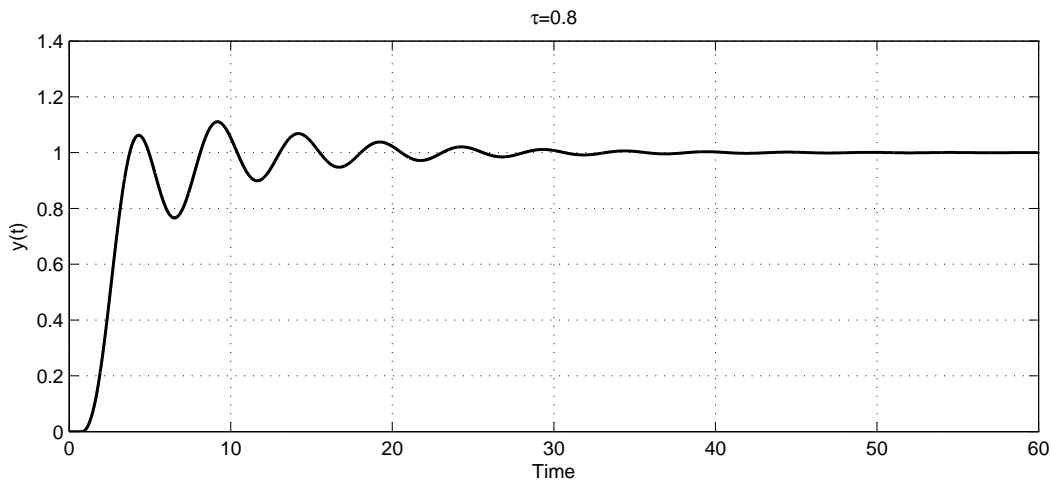

Figure 12: Closed Loop Performance

The scheme is simple and may be easily implemented: only four proportional gains and the model of the process are enough to stabilize the system. The procedure to design the controller can be performed easily using well know analysis of classical control theory. Numerical examples illustrate the performance of the controller under ideal conditions. The examples show the closed loop system behavior working under different initial conditions between the plant and the observer, as well as parametric variations. Moreover, the introduction of a PI controller allows to one to solve the problem of regulation and step disturbance rejection. Also, a robustness analysis is presented to show the effects of the modeling errors of the delay operator.

\section{Appendix A. Proof of The Lemma 2 .}

Let us consider the High-Order Unstable System with time delay given by:

$$
G(s)=\frac{\alpha}{(s-\sigma)\left(s+\phi_{1}\right)\left(s+\phi_{2}\right) \ldots\left(s+\phi_{n}\right)} e^{-\tau s} .
$$


First, analyzing the First Order Unstable Delayed System given by:

$$
G(s)=\frac{\alpha}{s-\sigma} e^{-\tau s}
$$

Considering the Lemma 1 there exists a proportional gain $k$ such that a closed loop system with a simple proportional output feedback is stable if and only if $\tau<\frac{1}{\sigma}$. An analysis in the frequency domain shall confirm this result. The Nyquist stability criteria establishes that, when closing the loop whit a proportional gain $k$, the system will be stable if $0=N+P$, with $P$ being the number of poles in the right half plane " $s$ " and $N$ the numbers of clockwise round trips to the point -1 ( $N$ negative suggests round trips in the opposite direction) in the Nyquist diagram. The angle as a function of the frequency $\omega$ is given by:

$$
\angle G(j \omega)=-\left(180^{\circ}-\arctan \left(\frac{\omega}{\sigma}\right)\right)-(\omega \tau)
$$

Taking into consideration that for small frequencies $\arctan \omega \varphi \approx \omega \varphi$, it can be shown that the condition $\tau<\frac{1}{\sigma}$ is equivalent to ask that the angle path taps at least one point (for some frequency) with a value exceeding $-180^{\circ}$, that is $\angle G(j \omega)>-180^{\circ}$, i.e., one counter-clockwise round trip to the point -1 in the Nyquist diagram. Now, analyzing the system with $n=1$ characterized by:

$$
G(s)=\frac{\alpha}{(s-\sigma)(s+\phi)} e^{-\tau s}
$$

Then, there exists a proportional gain $k$ such that a closed loop system whit a simple proportional output feedback is stable if and only if $\tau<\frac{1}{\sigma}-\frac{1}{\phi}$. It can be easy to see that the Nyquist condition remains the same (one counterclockwise round trip to the point -1 in the Nyquist diagram), now the angle condition is:

$$
\angle G(j \omega)=-\left(180^{\circ}-\arctan \left(\frac{\omega}{\sigma}\right)\right)-\left(\arctan \left(\frac{\omega}{\phi}\right)\right)-(\omega \tau)
$$

For small frequencies $\arctan \omega \varphi \approx \omega \varphi$, and starting from $\angle G(j \omega)>-180^{\circ}$ it is no difficult to conclude the relation $\tau<\frac{1}{\sigma}-\frac{1}{\phi}$. Below considering the system whit $n=2$ given by:

$$
G(s)=\frac{\alpha}{(s-\sigma)\left(s+\phi_{1}\right)\left(s+\phi_{2}\right)} e^{-\tau s} .
$$

It can be easy to see that the Nyquist condition remains the same (one counter-clockwise round trip to the point -1 in the Nyquist diagram), now the angle condition is:

$$
\begin{aligned}
\angle G(j \omega)= & -\left(180^{\circ}-\arctan \left(\frac{\omega}{\sigma}\right)\right)-\left(\arctan \left(\frac{\omega}{\phi_{1}}\right)\right)-\cdots \\
& \cdots-\left(\arctan \left(\frac{\omega}{\phi_{2}}\right)\right)-(\omega \tau)
\end{aligned}
$$


Again considering that for small frequencies $\arctan \omega \varphi \approx \omega \varphi$, and starting from $\angle G(j \omega)>-180^{\circ}$ it is no difficult to conclude the relation $\tau<\frac{1}{\sigma}-\frac{1}{\phi_{1}}-\frac{1}{\phi_{2}}$.

This reflection can be generalized to any $n \in \mathbb{R}$ concluding that for the systems characterized by (A.1) with a proportional output feedback, there exist a constant gain $k$ such that the closed loop system is stable if and only if:

$$
\tau<\frac{1}{\sigma}-\sum_{i=1}^{n} \frac{1}{\phi_{i}}
$$

\section{References}

[1] J.-P. Richard, Time-Delay Systems:An Overview of Some Recent Advances and Open Problems., Automatica 39 (2003) 1667-1694.

[2] Q.-C. Zhong, Robust Control of Time-Delay Systems, Springer, 2006.

[3] S. Skogestad, Simple Analytic Rules for Model Reduction and PID Controller Tunning, Journal of Process Control 13 (2003) 4.

[4] K. Gu, V. L. Kharitonov, J. Chen, Stability of Time-Delay Systems, Birkhäuser, 2003.

[5] S.-I. Niculescu, Delay Effects on Stability: A Robust Control Approach, Springer, 2001.

[6] Z. J. Palmor, Time delay compensation smith predictor and its modifications, The control Handbook (1996) 224- 237.

[7] O. J. M. Smith, Close Control of Loops with Dead Time, Chem. Eng. Prog. 53 (1957) 217-219.

[8] R. A. Seshagiri, V. S. R. Rao, M. Chidambaram, Simple analytical design of modified smith predictor with improved performance for unstable firstorder plus time delay (fodtp) processes, Ind. Eng. Chem. Res 46(13) (2007) $4561-4571$.

[9] J. E. Normey-Rico, E. F. Camacho, Dead-time compensators: A survey, Control Engineering Practice 16 (4) (2008) 407-428.

[10] J. E. Normey-Rico, E. F. Camacho, Unified approach for robust dead-time compesator design, Journal of Process Control 19 (2009) 38-47.

[11] A. Visioli, Q.-C. Zhong, Control of Integral Processes with Dead Time, Advances in Industrial Control, Springer London, 2011.

[12] G. J. Silva, A. Datta, S. Bhattacharyya., PID Controllers for Time-Delay Systems, Control Engineering, Birkhäuser, 2004. 
[13] R. H. Middleton, D. E. Miller., On the Achievable Delay Margin Using LTI Control for Unstable Plants, IEEE Transactions on Automatic Control 52 (7) (2007) 1194-1207.

[14] W. Michiels, K. Engelborghs, P. Vansevenant, D. Roose, Continuous pole placement for delay equations, Automatica 38 (5) (2002) $747-761$.

[15] B. del Muro Cuéllar, J. F. M. Rubio, M. Velasco-Villa, J. Álvarez Ramrez, On the control of unstable first order linear systems with large time lag: Observer based approach, European Journal of Control 5 (2012) in press.

[16] J. F. Márquez-Rubio, B. del Muro-Cuéllar, M. Velasco-Villa, D. F. NovellaRodríguez, Observer PID stabilization strategy for unstable first-order linear systems with large time delay, Industrial \& Engineering Chemistry Research 51 (25) (2012) 8477-8487.

[17] C. Madhuranthakam, A. Elkamel, H. Budman, Optimal tuning of PID controllers for foptd, soptd and soptd with lead processes, Chemical Engineering and Processing: Process Intensification 47 (2) (2008) $251-264$.

[18] A. S. Rao, M. Chidambaram[2], Enhanced Control of Unstable Cascade Processes with Time Delay Using a Modified Smith Predictor, Ind. Eng. Chem. Res. 48 (2009) 3098-3111.

[19] A. S. Rao, M. Chidambaram, Control of unstable processes with two rhp poles, a zero and time delay, Asia-Pacific Journal of Chemical Engineering 1 (1-2) (2006) 63-69.

[20] M. Shamsuzzoha, M. Skliar, M. Lee, Design of IMC filter for PID control strategy of open-loop unstable processes with time delay, Asia-Pacific Journal of Chemical Engineering 7 (1) (2012) 93-110.

[21] B. Del-Muro-Cuéllar, J. Márquez-Rubio, M. Velasco-Villa, J. AlvarezRámirez, Stabilisation and control of unstable first-order linear delay systems, Int. J. of Computer Applications in Technology 41 (1/2) (2011) 84-90.

[22] E. Malakhovski, L. Mirkin, On Stability of Second-order Quasi-polynomials with a Single Delay, Automatica 42 (2006) 1041-1047.

[23] S. C. Lee, Q.-G. Wang, C. Xiang, Stabilization of all-pole unstable delay processes by simple controllers, Journal of Process Control 20 (2) (2010) $235-239$.

[24] K. J. Astrom, T. Hagglund, PID Controllers: Theory, Design, and Tuning, 2nd Edition, ISA International, 1995.

[25] E. I. Verriest, O. Sename, P. Pepe, Robust Observer-Controller for Delay Differential Systems, Procedings of the 41st IEEE Conference on Decision and Control (2002) 981-986. 
[26] O. Sename, Is a Mixed Design of Observer Controllers for Time Delay Systems Interesting?, Asian Journal of Control 9 (2) (2007) 180-189.

[27] B. W. Bequette, Process Control. Modeling, Design and Simulation, Prentice Hall Internacional, 2003. 